\title{
Relationship between biological behavior and phenotypic expression in undifferentiated-type gastric carcinomas
}

\author{
Akira Kabashima ${ }^{1,2}$, Takashi YaO $^{1}$, Yoshihiko Maehara ${ }^{2}$, and Masazumi Tsuneyoshi ${ }^{1}$ \\ ${ }^{1}$ Department of Anatomic Pathology, Graduate School of Medical Sciences, Kyushu University, 3-1-1 Maidashi, Higashi-ku, \\ Fukuoka 812-8582, Japan \\ ${ }^{2}$ Department of Surgery and Sciences, Graduate School of Medical Sciences, Kyushu University, Fukuoka, Japan
}

\begin{abstract}
Background. It has been proved that some differentiated-type gastric carcinomas have a gastric phenotype. Similarly, it can be conjectured that some undifferentiated-type gastric carcinomas have an intestinal phenotype and that there are biological differences between undifferentiated-type gastric carcinomas with a gastric phenotype and those with an intestinal phenotype. We classified the phenotypes of early undifferentiated-type gastric carcinomas and investigated the relationship between their biological behavior and the phenotypes.

Methods. Sixty lesions of intramucosal undifferentiated-type gastric carcinoma were classified into four phenotypes; gastric type, incomplete-intestinal type, complete-intestinal type, and unclassified type, according to the expression of CD10, MUC2, small-intestinal mucinous antigen (SIMA), human gastric mucin (HGM), or concanavalin A (ConA).

Results. The incidence of gastric-type carcinoma, incompleteintestinal-type carcinoma, and complete-intestinal-type carcinoma was $33 \%$ ( 20 cases), $65 \%$ (39 cases), and $2 \%$ (1 case), respectively. There was no significant difference in any of the clinicopathological factors examined between the 20 gastrictype carcinomas and the 40 intestinal-type carcinomas, but there were significant differences in the morphological findings. Intestinal-type carcinomas had a glandular structure more frequently than the gastric-type carcinomas. The spreading pattern of gastric-type carcinomas showed a middle-layer type more frequently than the intestinal-type carcinomas.

Conclusion. Undifferentiated-type gastric carcinomas frequently expressed an intestinal phenotype. There were differences in the growth patterns between undifferentiated-type gastric carcinomas with a gastric phenotype and those with the intestinal phenotype.
\end{abstract}

Key words Phenotypic expression · Undifferentiated-type gastric carcinomas $\cdot$ Growth pattern

Offprint requests to: $\mathrm{T}$. Yao

Received: December 10, 2004 / Accepted: June 15, 2005

\section{Introduction}

Generally, gastric carcinomas are classified into two histological types by standard hematoxylin and eosin (H\&E) staining; such as "intestinal" type and "diffuse" type, by Lauren [1], and "differentiated" type and "undifferentiated" type by Nakamura et al. [2]. It has been considered that intestinal-type carcinoma is similar to differentiated-type carcinoma and that diffuse-type carcinoma and undifferentiated-type carcinoma are similar and show a gastric phenotype. However, there are currently various opinions regarding the classification of gastric carcinoma phenotypes by mucin-histochemical or immunohistochemical methods. The existence of differentiated-type gastric carcinomas having a gastric phenotype has been proved. Tatematsu et al. [3] and Egashira [4] reported that about $30 \%$ of differentiatedtype gastric carcinomas showed a gastric phenotype, by immunohistochemical or mucin-histochemical studies. We have reported that, in immunohistochemical studies, $38.8 \%$ of differentiated-type gastric carcinomas showed a gastric phenotype [5]. Generally, differentiated-type gastric carcinomas with a gastric phenotype are considered to have high invasiveness and high metastatic potential compared with differentiated-type gastric carcinomas with an intestinal phenotype [6-11]. It has been reported that there are undifferentiatedtype gastric carcinomas having an intestinal phenotype $[12,13]$. Fiocca et al. [14] and Yamachika et al. [15] classified the phenotypes of gastric signet-ring cell carcinomas, and reported that the progression of gastric carcinomas was associated with a phenotypic shift from gastric type to intestinal type. Similarly, Yao et al. [16] reported that intestinalization frequently occurred during neoplastic transformation. It can be conjectured that there are biological differences between undifferentiated-type gastric carcinomas with a gastric phenotype and those with an intestinal phenotype. But there has been no report about comparisons of biological behav- 
ior between undifferentiated-type gastric carcinomas having a gastric phenotype and those having an intestinal phenotype.

In the study, we report here, we classified the phenotypes of early undifferentiated-type gastric carcinomas according to the type of intestinal metaplasia [5] and investigated the relationship between their biological behavior and the phenotypes.

\section{Materials and methods}

\section{Materials}

From our pathological files of gastric specimens that had been surgically resected at Kyushu University Hospital and its affiliated hospitals, 60 lesions of intramucosal undifferentiated-type gastric carcinoma were randomly selected for the present study. Undifferentiated-type gastric carcinoma was defined as poorly differentiated adenocarcinoma or signet-ring cell carcinoma. All the lesions were cut into serial step sections of $3-4 \mathrm{~mm}$ in width, fixed in $10 \%$ formalin solution, and embedded in paraffin. Macroscopic and histological evaluations were made according to the classification established by the Japanese Research Society for Gastric Cancer [17]. The macroscopic features were divided into three major types; type I (polypoid), type II (superficial), and type III (excavated). Type II was further divided into three subtypes: type IIa (elevated), type IIb (flat), and type IIc (depressed). Composite types were classified based on the predominant subtype; for example, type IIc+III was considered to be type IIc. The depth of invasion and histological grade were classified according to the predominant component.

\section{Immunohistochemical staining}

To classify the phenotypic expression, the expressions of CD10, MUC2, small intestinal mucinous antigen (SIMA), human gastric mucin (HGM), or concanavalin A (ConA) were investigated by immunohistochemical methods. We consider that CD10, MUC2, and SIMA are detected in the intestinal phenotype, while HGM and ConA are detected in the gastric phenotype. The staining of CD10, MUC2, HGM, and ConA was carried out according to previous studies [5]. The staining of SIMA was carried out by the streptavidin-biotin (SAB) method with an antibody against SIMA (SIMA-4D3; NovoCastra, Newcastle, England) and SAB-PO (mouse) kits (Nichirei, Tokyo, Japan). Sections (4- $\mu$ mthick) were deparaffinized in xylene, hydrated through a graded series of ethanol, and immersed in 3\% hydrogen peroxide, followed by immersion in $100 \%$ methanol for $30 \mathrm{~min}$ to inhibit endogenous peroxidase. To activate the antigens, the slides were placed in a microwave oven in $10 \mathrm{mM}$ citrate buffer ( $\mathrm{pH} 6.0$ ) for $30 \mathrm{~min}$. After a rinsing in phosphate-buffered saline (PBS), the slides were incubated in humid chambers with the primary antibodies, SIMA at 1:100, overnight at $4{ }^{\circ} \mathrm{C}$, followed by three washes with PBS. The sections were then incubated with biotinylated anti-mouse immunoglobulin (Ig) G, IgA, and IgM for $20 \mathrm{~min}$, and with peroxidaseconjugated streptavidin for $20 \mathrm{~min}$. After being washed in PBS, the slides were developed by being immersed in $0.01 \% \mathrm{H} 2 \mathrm{O} 2$ and $0.05 \%$ diaminobenzidine tetrahydrochloride (DAB) for $3 \mathrm{~min}$. A light counterstaining with Mayer's hematoxylin was carried out. The results of staining were categorized into two groups: positive expression and negative expression. When more than $10 \%$ of the carcinoma cells in the neoplastic areas were stained, it was classified as positive expression. When fewer than $10 \%$ of the carcinoma cells in the neoplastic areas were stained, it was classified as negative expression.

\section{Classification and comparisons of phenotypic expression}

Classification of the phenotypes of the carcinomas and the background mucosas was based on the classification of intestinal metaplasia (complete-type intestinal metaplasia, incomplete-type intestinal metaplasia, or nonmetaplastic gastric mucosa) by immunohistochemical staining, according to previous studies [5,18]. The phenotypes were classified into four groups; gastric type, incomplete-intestinal type, complete-intestinal type, and unclassified type, according to the combination of the expression of CD10, MUC2, SIMA, HGM, or ConA. Figure 1 summarizes the classification of the

\begin{tabular}{|c|c|c|c|}
\hline & & $\begin{array}{c}\mathrm{HGM} \text { or ConA } \\
(+)\end{array}$ & $\begin{array}{l}\text { HGM and ConA } \\
(-)\end{array}$ \\
\hline \multicolumn{2}{|l|}{$\mathrm{CD} 10(+)$} & \multirow{2}{*}{$\begin{array}{l}\text { Incomplete } \\
\text { intestinal-type }\end{array}$} & $\begin{array}{l}\text { Complete } \\
\text { intestinal-tyne }\end{array}$ \\
\hline \multirow{2}{*}{ CD10(-) } & $\begin{array}{c}\text { MUC2 or SIMA } \\
(+)\end{array}$ & & \\
\hline & $\begin{array}{c}\text { MUC2 and SIMA } \\
(-)\end{array}$ & Gastric-type & Unclassified-type \\
\hline
\end{tabular}

Fig. 1. Classification of phenotypes. The phenotypes were classified into four groups; gastric type, incomplete-intestinal type, complete-intestinal type, and unclassified type, according to the combination of the expression of CD10, MUC2, small-intestinal mucinous antigen $(S I M A)$, human gastric mucin $(H G M)$, or concanavalin A $(\operatorname{Con} A)$ 
Middle layer type

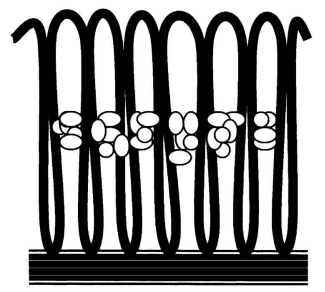

Whole layer type
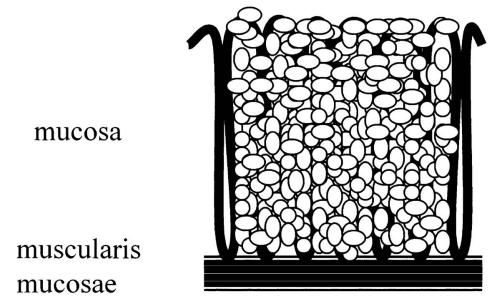

Fig. 2. Spreading features. The spreading features were classified into two patterns, the middle-layer type and the whole-layer type. In the middle-layer type, the carcinoma cells mainly spread in the gastric mucosa between the proper foveolae. In the whole-layer type, the carcinoma cells mainly expand in the gastric mucosa with destruction of the foveolae

phenotypes of the carcinomas and the background mucosas. We compared the clinicopathological findings and the morphological findings (including the existence of glandular structures or signet-ring cells and the spreading pattern) between gastric-type carcinomas and intestinal-type carcinomas (including incompleteintestinal-type carcinomas and complete-intestinal-type carcinomas). The spreading patterns were classified into two types, middle-layer type and whole-layer type, as shown in Fig. 2.

\section{Statistical analyses}

The BMDP statistical package program (BMDP; Los Angeles, CA, USA) for an IBM (Armonk, NY, USA) 4381 mainframe computer was used for all analyses. The relationships between the clinicopathological findings or the morphological findings and the phenotypes were examined by the $\chi^{2}$ test and Kruskal-Wallis test. The level of significance was less than 0.05 .

\section{Results}

\section{Clinicopathological findings}

Gastric-type carcinoma is shown in Fig. 3. This carcinoma showed the middle-layer-type spreading pattern. The upper layer of the carcinoma was positive for HGM, while the lower layer of the carcinoma was positive for ConA, as was the normal layer structure of gastric proper mucosa. Incomplete-intestinal-type carcinoma is shown in Fig. 4. This carcinoma showed the whole-layer-type spreading pattern. This carcinoma was negative for HGM and ConA, and positive for SIMA. Complete-intestinal-type carcinoma is shown in Fig. 5. This carcinoma had structures of small trabeculae and microglands. This carcinoma was negative for HGM
Table 1. Clinicopathological findings

\begin{tabular}{lccc}
\hline Carcinoma & $\begin{array}{c}\text { Gastric type } \\
(n=20)\end{array}$ & $\begin{array}{c}\text { Intestinal type } \\
(n=40)\end{array}$ & \\
\hline Sex (male:female) & $10: 10$ & $20: 20$ & NS \\
Age (average, years) & $54.4 \pm 9.1$ & $55.5 \pm 11.5$ & NS \\
Location & 1 & & NS \\
$\quad$ C & 12 & 22 & \\
M & 7 & 16 & \\
A & 2 & 2 & NS \\
Gross classification & 18 & 37 & \\
$\quad$ IIb & 0 & 1 & \\
IIc & & $3.5 \pm 1.8$ & NS \\
III & & & \\
Size (average, cm) & $2.8 \pm 1.7$ & &
\end{tabular}

and ConA, while the laminar surface of the carcinoma was positive for CD10. The incidence of gastric-type carcinomas, incomplete intestinal-type carcinomas, and complete intestinal-type carcinomas was 33\% (20 cases), 65\% (39 cases), and 2\% (1 cases), respectively.

Table 1 shows the clinicopathological findings of the 20 gastric-type carcinomas and 40 intestinal-type carcinomas (incomplete-intestinal-type carcinomas and complete-intestinal-type carcinomas). Intestinal-type carcinomas tended to be larger than gastric-type carcinomas, but there were no significant differences in any clinicopathological factors examined between gastrictype carcinomas and intestinal-type carcinomas.

\section{Morphological findings}

Table 2 shows the morphological findings of the 20 gastric-type carcinomas and the 40 intestinal-type carcinomas. Intestinal-type carcinomas had a glandular structure more frequently than gastric-type carcinomas. Gastric-type carcinomas had the middle-layer type spreading pattern more frequently than intestinal-type carcinomas. On the other hand, intestinal-type carcinomas had the whole-layer type spreading pattern more frequently than gastric-type carcinomas. In addition, the whole-layer-type carcinomas tended to be larger than the middle-layer-type carcinomas (average size, $3.3 \mathrm{~cm}$ vs $2.8 \mathrm{~cm})$.

\section{Relationship between carcinoma phenotype and that of} the background muсоsa

Table 3 shows the relationship between the phenotype of the carcinomas and that of the background mucosa. The phenotype of the carcinomas was significantly related to that of their own background mucosa $(P=$ $0.0440)$. The spreading pattern of gastric-type carcino- 


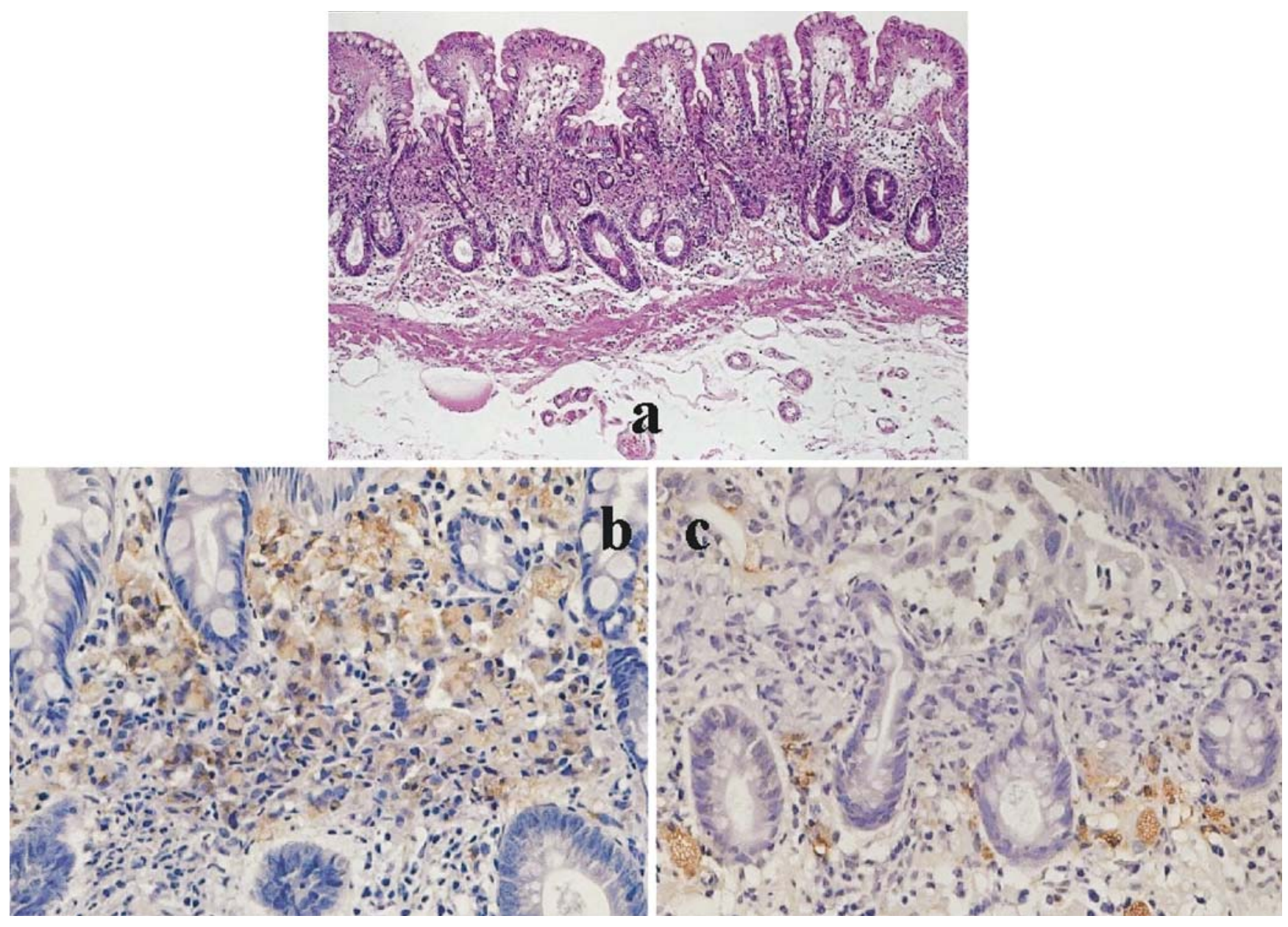

Fig. 3a-c. Gastric-type carcinoma. a This carcinoma shows middle-layer type spreading and arises in incomplete-intestinal-type background mucosa. $\times 50$. b The upper layer of the carcinoma is positive for HGM. $\times 100$. $\mathbf{c}$ The lower layer of the carcinoma is positive for ConA. $\times 100$

Table 2. Morphological findings

\begin{tabular}{lccc}
\hline Carcinoma & $\begin{array}{c}\text { Gastric type } \\
(n=20)\end{array}$ & $\begin{array}{c}\text { Intestinal type } \\
(n=40)\end{array}$ & \\
\hline $\begin{array}{l}\text { Glandular structure* } \\
(-)\end{array}$ & 16 & 17 & $P=0.0059$ \\
$\quad(+)$ & 4 & 23 & \\
$\begin{array}{l}\text { Signet-ring cell } \\
(-)\end{array}$ & 5 & 20 & $P=0.0641$ \\
$\quad(+)$ & 15 & 20 & \\
Spreading pattern* & & & \\
$\quad$ Middle-layer type & 12 & 46 & \\
$\quad$ Whole-layer type & 8 & 36 & \\
\hline
\end{tabular}

* Significant

mas tended to be the middle-layer type and the spreading pattern of intestinal-type carcinomas tended to be the whole-layer type, regardless of the phenotype of their own background mucosa.

\section{Discussion}

In this study, we classified the phenotypes of undifferentiated-type gastric carcinomas, based on the expression of CD10, MUC2, SIMA, HGM, or ConA 


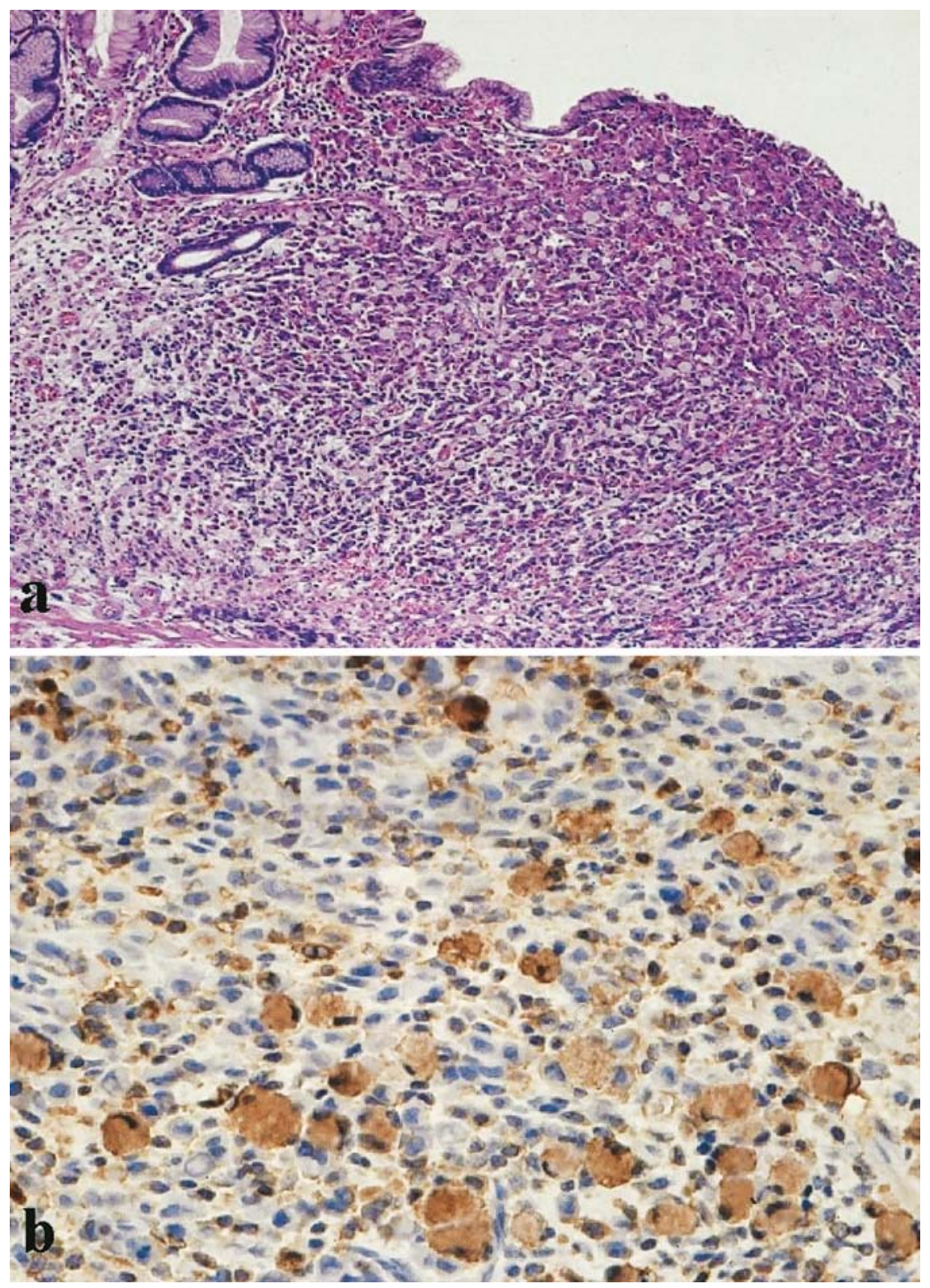

Fig. 4a,b. Incomplete-intestinal-type carcinoma. a This carcinoma shows wholelayer-type spreading pattern. $\times 50$. b This carcinoma is positive for SIMA. $\times 100$

Table 3. Relationship between the phenotype of the carcinomas and that of the background mucosa

\begin{tabular}{lccc}
\hline Carcinoma & $\begin{array}{c}\text { Gastric type } \\
(n=20)\end{array}$ & $\begin{array}{c}\text { Intestinal type } \\
(n=40)\end{array}$ & \\
\hline Background mucosa & & & $P=0.0440$ \\
$\quad$ Gastric type & $13(\mathrm{M}, 8 ; \mathrm{W}, 5)$ & $15(\mathrm{M}, 2 ; \mathrm{W}, 13)$ & \\
$\quad$ Intestinal type & $7(\mathrm{M}, 4 ; \mathrm{W}, 3)$ & $25(\mathrm{M}, 2 ; \mathrm{W}, 23)$ & \\
\hline
\end{tabular}

$\mathrm{M}$, middle-layer-type carcinoma; w, whole-layer-type carcinoma

and the classification of intestinal metaplasia. In previous studies, it was shown that CD10, MUC2, HGM, and ConA were expressed in the brush border, the goblet cells, the gastric foveolar epithelium, and the pyloric glands, individually [19-26]. We employed SIMA, for the first time, for the evaluation of intestinal phenotypic expression. SIMA is a $1000-\mathrm{kDa}$ mucin glycoprotein antigen that is known to be present in the goblet cells and the extracellular mucin of the small intestine. SIMA is not present in normal stomach and normal adult large 


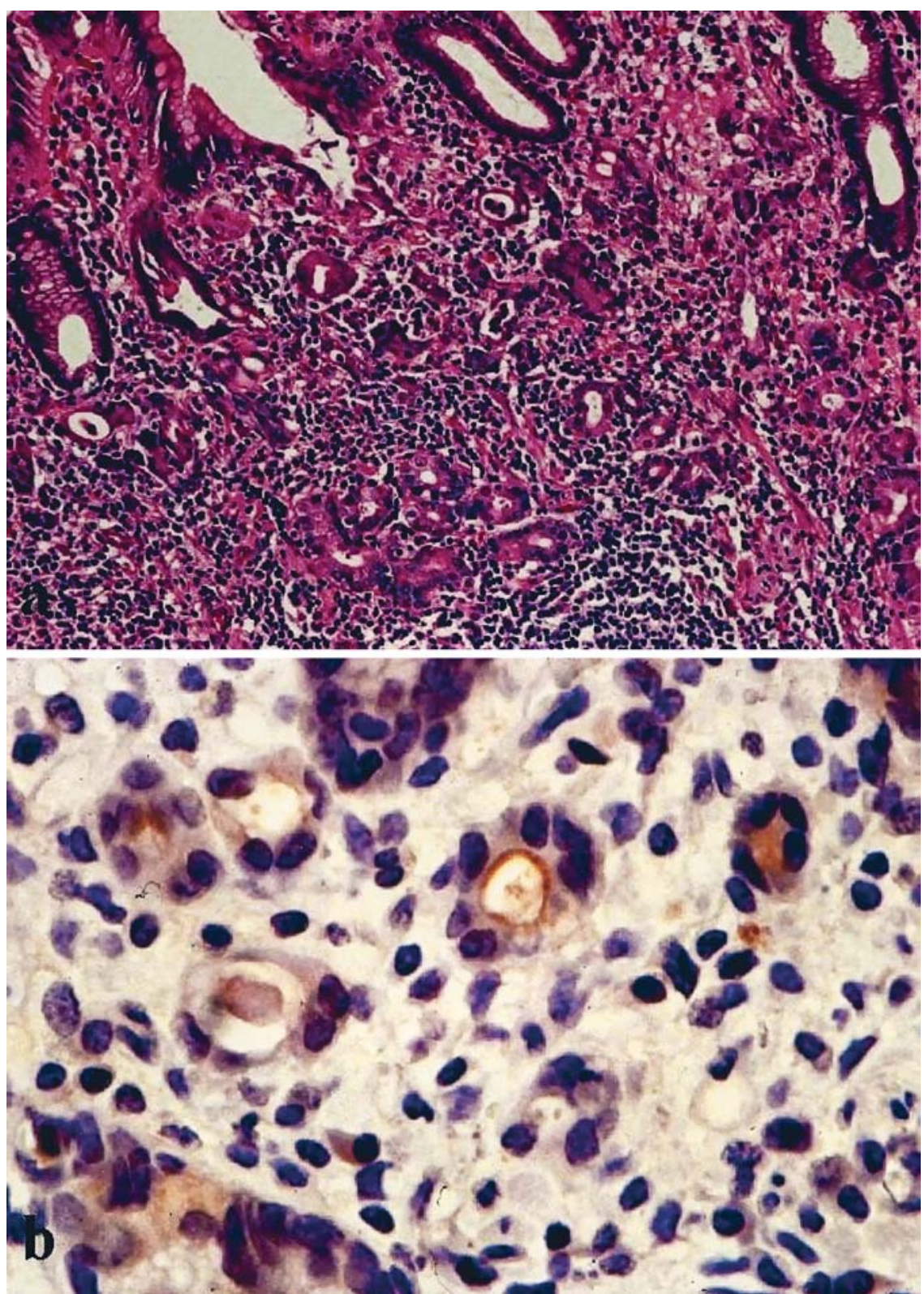

Fig. 5a,b. Complete-intestinal-type carcinoma. a This carcinoma has structures of small trabeculae and microglands. $\times 50$. b The laminar surface of this carcinoma is positive for CD10. $\times 100$ bowel. But SIMA is reported to be present in cancers of the stomach and large bowel [27-30]. In this study, 67\% of undifferentiated-type gastric carcinomas had an intestinal phenotype. Sepulveda et al. [31] reported that $46 \%$ of undifferentiated-type gastric carcinomas had an intestinal phenotype. Fujimori et al. [32] reported diffuse-type gastric carcinomas that were composed of Paneth-cell-type carcinoma cells. Wang et al. [33] reported diffuse-type gastric carcinomas that expressed human colonic mucin (HCM14 or HCM21).

In differentiated-type gastric carcinomas, it was reported that intramucosal carcinomas tended to have a phenotype similar to the phenotype of the background mucosa. It has also been demonstrated that even intramucosal diffuse-type gastric carcinomas have the laminated structure of mucins similar to that in the background mucosa [34-35]. In the present study, several carcinomas were positive for the foveolar epithelial marker (HGM) in the upper layer and positive for the pyloric glandular marker (ConA) in the lower layer, similar to the layer structure of the gastric proper mucosa. Also, the phenotype of the carcinomas tended to imitate the phenotype of their own background mucosa, as reported in previous studies. It is considered that intramucosal carcinomas, even those of the undifferentiated type, tend to keep the phenotype of the background mucosa. Solcia et al. [36] reported that there were two carcinogenetic pathways of diffuse-type gastric carcinoma. We also consider that undifferentiatedtype gastric carcinomas have a carcinogenetic pathway 
not only from the gastric proper mucosa but also a carcinogenetic pathway from intestinal metaplasia. We considered that, as gastric carcinomas increased in size, the whole-layer type was shown, and that the progression of carcinomas was associated with a phenotypic shift from gastric-type expression to intestinal-type expression, as Yamachika et al. [15] and Yao et al. [16] have reported.

In this study, there were no clinicopathological differences between gastric-type carcinomas and intestinaltype carcinomas, but there were morphological differences. Gastric-type carcinomas more commonly had the middle-layer type, spreading pattern compared with intestinal-type carcinomas. In a carcinoma with the middle-layer type, spreading pattern the tumor margins tend to be unclear, because the carcinoma cells do not appear at the surface of the mucosa. So the margin of gastric-type carcinoma is considered to be unclear. Similarly, in differentiated-type carcinomas, Yoshino et al. [6] reported that the margin of gastric-type carcinoma was unclear compared with that in intestinal-type carcinoma. For gastric-type carcinomas, in particular the area to be resected and the choice of endoscopic mucosal resection (EMR) has to be carefully considered.

Our immunohistochemical method can be used to classify the phenotypes of gastric carcinomas, without regard to the presence or absence of a glandular structure, and we have shown that undifferentiated-type carcinomas frequently express an intestinal phenotype. There were differences in the growth patterns between undifferentiated-type gastric carcinomas with a gastric phenotype and those with the intestinal phenotype.

\section{References}

1. Lauren P. The two histological main types of gastric carcinoma: diffuse and so-called intestinal-type carcinoma. Acta Pathol Microbiol Immunol Scand 1965;64:31-49.

2. Nakamura K, Sugano H, Takagi K. Carcinoma of stomach in incipient phase: its histogenesis and histological appearances. GANN 1968;59:251-8.

3. Tatematsu M, Ichinose M, Miki K, Hasegawa R, Kato T, Ito N. Gastric intestinal phenotypic expression of human stomach cancers as revealed by peptinogen immunohistochemistry and mucin histochemistry. Acta Pathol Jpn 1990;40:494-504.

4. Egashira Y. Mucin histochemical study of differentiated adenocarcinoma of stomach. Jpn J Gastroenterol 1994;91:839-48.

5. Kabashima A, Yao T, Tsuneyoshi M. Gastric and intestinal phenotypic expression in the carcinomas and background mucosa of multiple early gastric carcinomas. Histopathology 2000;37:51322.

6. Yoshino T, Shimoda T, Saitou A, Nakanishi Y, Tajima Y, Shirasu $\mathrm{T}$, et al. Macroscopic features of differentiated gastric adenocarcinoma with gastric or intestinal phenotype expression in early gastric cancer. I to Cho (Stomach and Intestine) 1999;34:507-25.

7. Kubo K, Yanagisawa A, Ninomiya $\mathrm{Y}$, Inoshita N, Kato $\mathrm{Y}$. Characteristics of differentiated-type carcinoma with gastric phenotype of the stomach. I to Cho (Stomach and Intestine) 1999;34:487-94.

8. Koseki K, Takizawa T, Koike M, Funata N, Hishima T, Sakoma $\mathrm{R}$, et al. Subclassification of well differentiated gastric cancer with reference to biological behavior and malignancy, gastric type vs intestinal type, and papillary carcinoma vs tubular carcinoma. I to Cho (Stomach and Intestine) 1999;34:507-12.

9. Nishikura K, Watanabe H, Ninomiya Y, Ajioka Y, Hashidate H, et al. Differentiated gastric adenocarcinoma with gastric phenotype. Its new classification and histopathological characteristics. I to Cho (Stomach and Intestine) 1999;34:495-506.

10. Endou Y, Tamura G, Sakata K, Oomura K, Watanabe H, Motoyama T. Genetic analysis of differentiated-type adenocarcinomas of the stomach with gastric phenotype and intestinal phenotype. I to Cho (Stomach and Intestine) 1999;34:539-44.

11. Oya M, Yao T, Tsuneyoshi M. A correlation of intramural invasion with mucin histochemistry, and immunohistochemical expressions of oncogene-related proteins in early gastric carcinoma. I to Cho (Stomach and Intestine) 1997;32:31-9.

12. Fiocca R, Villani L, Tenti P, Cornaggia M, Finzi D, Capella C, et al. Widespread expression of intestinal markers in gastric carcinoma: a light and electron microscopic study using BD-5 monoclonal antibody. J Clin Pathol 1988;41:178-87.

13. Fiocca R, Villani L, Tenti P, Cornaggia M, Finzi D, Riva C, et al. The foveolar cell component of gastric cancer. Hum Pathol 1990; 21:260-70.

14. Fiocca R, Villani L, Tenti P, Cornaggia M, Frigerio B, Solcia E, et al. Characterization of four main cell types in gastric cancer: foveolar, mucopeptic, intestinal columnar and goblet cells. Pathol Res Pract 1987;182:308-25.

15. Yamachika T, Inada K, Fujimitu Y, Nakamura S, Yamamura Y, Kitou $\mathrm{T}$, et al. Intestinalization of gastric signet ring cell carcinomas with progression. Virchows Arch 1997; 431: 103-10.

16. Yao T, Kajiwara M, Kuroiwa S, Iwashita A, Oya M, Kabashima A, et al. Malignant transformation of gastric hyperplastic polyps: alteration phenotypes, proliferative activity, and p53 expression. Hum Pathol 2002;33:1016-22.

17. Japanese Research Society for Gastric Cancer. The general rules for gastric cancer study, 12th ed. Tokyo: Kinbara; 1993.

18. Yao T, Kabashima A, Kouzuki T, Oya M, Tsuneyoshi M. The phenotypes of the gastric carcinoma. Evaluation by a new immunohistochemical method. I to Cho (Stomach and Intestine) 1999:34:477-85.

19. Danielsen EM, Vyas JP, Kenny AJ. A neutral endopeptidase in the microvillar membrane of pig intestine. Biochem J 1980;191: 645-8.

20. Trejdosiewicz LK, Malizia G, Oakes J, Losowsky MS, Janossy G. Expression of the common acute lymphoblastic leukemia antigen in the brush border of normal jejunum and jejunum of patients with coeliac disease. J Clin Pathol 1985;38:1002-6.

21. Endoh Y, Tamura G, Motoyama T, Ajioka Y, Watanabe H. Welldifferentiated adenocarcinoma mimicking complete-type intestinal metaplasia in the stomach. Hum Pathol 1999;30:826-32.

22. Tytgat KMAJ, Buller HA, Opdam FJM. Biosynthesis of human colon mucin: MUC2 is prominent secretory mucin. Gasteroenterology 1994;107:1352-63.

23. Ajioka Y, Watanabe H, Jass JR. MUC1 and MUC2 mucin in flat and polypoid colorectal adenomas. J Clin Pathol 1997;50:417-21.

24. Ajioka Y, Allison LJ, Jass JR. Significance of MUC1 and MUC2 mucin expression in colorectal cancer. J Clin Pathol 1996;49:5604.

25. Bara J, Gautier R, Zaghouani H, Decaens C, Daher N. Monoclonal antibodies against oncofetal mucin M1 antigens associated with precancerous colonic mucosae. Cancer Res 1986;46:3983-9.

26. Bara J, Gautier R, Mouradian P, Decaens C, Daher N. Oncofetal mucin M1 epitope family: characterization and expression during colonic carcinogenesis. Int J Cancer 1991;47:304-10.

27. Hertzog PJ, Robinson HC, Ma J, Mackay IR, Linnane AW. Oncofetal expression of the human intestinal mucin glycoprotein 
antigens in gastrointestinal epithelium defined by monoclonal antibodies. Int J Cancer 1991;48:355-63.

28. Pilbrow SJ, Hertzog PJ, Linnane AW. The adenoma-carcinoma sequence in the colorectum - early appearance of a hierarchy of small intestinal mucin antigen (SIMA) epitopes and correlation with malignant potential. Br J Cancer 1992;66:748-57.

29. Pilbrow SJ, Hertzog PJ, Linnane AW. Differentiation-associated changes in mucin glycoprotein antigenicity in mucosa adjacent to rare gastrointestinal tract tumors of non-mucosal origin. J Pathol 1993;169:259-67.

30. Pinczower GD, Williams RPW, Gianello RD, Robinson HC, Preston BN, Linnane AW. Characterisation of the tumorassociated carbohydrate epitope recognized by monoclonal antibody 4D3. Int J Cancer 1996;66:636-44.

31. Sepulveda AR, Wu L, Ota H, Gutierrrez O, Kim JG, Genta RM, et al. Molecular identification of main cellular lineages as a tool for the classification of gastric cancer. Hum Pathol 2000;31:56674.

32. Fujimori Y, Akamatsu T, Ota H, Katsuyama T. Proliferative markers in gastric carcinoma and organoid differentiation. Hum Pathol 1995;26:725-34.
33. Wang YZ, Mitomi H, Kurihara M, Ishihara K, Hotta K, Tanigawa $\mathrm{H}$, et al. Gastric adenomas and superficial adenocarcinomas display distinct patterns of mucin carbohydrate and core protein expression. Histopathology 2000;37:250-9.

34. Akamatsu T, Katsuyama T. Histochemical demonstration of mucins in the intramucosal laminated structure of human gastric signet ring carcinoma and its relation to submucosal invasion. Histochem J 1990;22:416-25.

35. Sugihara H, Hattori T, Fukuda M, Fujita S. Cell proliferation and differentiation in intramucosal and advanced signet ring cell carcinomas of human stomach. Virchows Arch A 1987;411:11727.

36. Solcia E, Fiocca R, Luinetti O, Villani L, Padovan L, Calistri D, et al. Intestinal and diffuse gastric cancers arise in a different background of Helicobacter pylori gastritis through different gene involvement. AJSP 1996;20:8-22. 